\title{
La partición de Kosovo: ¡redibujar fronteras para conseguir la paz?
}

\author{
Miguel Borja Bernabé-Crespo
}

Universidad de Murcia. Departamento de Geografía

miguelborja.bernabe@um.es

\section{Resumen}

Kosovo es un Estado parcialmente reconocido con una gran variedad de minorías étnicas, donde la relación albanesa-serbia es la que ocasiona más tensiones. La propuesta de intercambio de territorios para corregir la frontera entre Serbia y Kosovo presenta como beneficio el reconocimiento mutuo y la normalización de relaciones, pero entraña el riesgo de abrir la caja de Pandora de las revisiones fronterizas en los Balcanes. El artículo incluye un repaso de las relaciones entre Serbia y Kosovo con una abundante selección de notas de prensa. Analiza dicha propuesta y refleja la situación actual de los territorios y debate su idoneidad. Concluye que la UE debería ofrecer claridad y un compromiso firme y tangible de progreso para afianzar su credibilidad, a la vez que apoyar la democratización para favorecer su integración europea.

Palabras clave: corrección de fronteras; minorías étnicas; intercambio de territorios; Balcanes Occidentales; Kosovo

\section{Resum. La partició de Kosovo: redibuixar fronteres per aconseguir la pau?}

Kosovo és un estat parcialment reconegut amb una gran varietat de minories ètniques, on la relació albanesa-sèrbia és la que ocasiona més tensions. La proposta d'intercanvi de territoris per corregir la frontera entre Sèrbia i Kosovo presenta com a benefici el reconeixement mutu i la normalització de relacions, però comporta el risc d'obrir la capsa de Pandora de les revisions frontereres als Balcans. L'article inclou un repàs de les relacions entre Sèrbia i Kosovo amb una abundant selecció de notes de premsa. Analitza aquesta proposta i reflecteix la situació actual dels territoris i debat la seva idoneïtat. Conclou que la UE hauria d'oferir claredat i un compromís ferm i tangible de progrés per consolidar la seva credibilitat, i alhora donar suport a la democratització per afavorir la seva integració europea.

Paraules clau: correcció de fronteres; minories ètniques; intercanvi de territoris; Balcans Occidentals; Kosovo 
Résumé. La partition du Kosovo : redessiner les frontières pour parvenir à la paix?

Le Kosovo est un État partiellement reconnu avec une grande variété de minorités ethniques, les relations albano-serbes provoquant le plus de tensions. L'échange de territoires proposé pour corriger la frontière entre la Serbie et le Kosovo présente la reconnaissance mutuelle et la normalisation des relations comme des avantages, mais comporte le risque d'ouvrir la boîte de Pandore de réajustements des frontières dans les Balkans. L'article présente une analyse des relations entre la Serbie et le Kosovo avec une sélection abondante de communiqués de presse. Il examine ladite proposition et reflète la situation actuelle des territoires tout en discutant leur pertinence. Il conclut que l'UE doit apporter de la clarté et un engagement ferme et tangible pour progresser afin de renforcer sa crédibilité, ainsi que de soutenir la démocratisation pour favoriser leur intégration européenne.

Mots-clés : correction des frontières; minorités ethniques; échange de territoires; Balkans occidentaux; Kosovo

\section{Abstract. The partition of Kosovo: Redrawing borders to achieve peace?}

Kosovo is a partially recognized state with a wide variety of ethnic minorities, with the Albanian-Serbian relationship causing the most tensions. The proposal to exchange territories (land swap) in order to correct the border between Serbia and Kosovo has the benefit of mutual recognition and normalization of relations, but entails the risk of opening up Pandora's box of border adjustments in the Balkans. The article includes a review of the relations between Serbia and Kosovo with a broad selection of press releases. It analyzes the proposal and reflects on the current situation of the territories and discusses its suitability. The article concludes that the EU should provide greater clarity and make a firm and tangible commitment toward progress to strengthen its credibility, as well as support democratization to promote European integration.

Keywords: border change; ethnic minorities; land swap; Western Balkans; Kosovo

\section{Sumario}

1. Introducción

2. Contexto histórico: del mito de Kosovo a la declaración de independencia y el diálogo de Bruselas

3. La situación actual en Kosovo del Norte y Valle de Preševo
4. Propuesta de intercambio de territorios

5. Discusión

6. Conclusiones

Referencias bibliográficas

\section{Introducción}

La traumática desintegración yugoslava no solo causó un gran número de víctimas y enormes daños materiales, sino que también dejó a la sociedad dividida a lo largo de líneas étnicas. En gran parte debido al ascenso de movimientos nacionalistas derivados de las hostilidades de los diferentes grupos étnicos (Blitzt, 2006; Ramet, 2002), actualmente siguen existiendo tensiones entre antiguos adversarios, máxime en Kosovo, un territorio parcialmente reconocido 
Mapa 1. Contexto de la ampliación europea

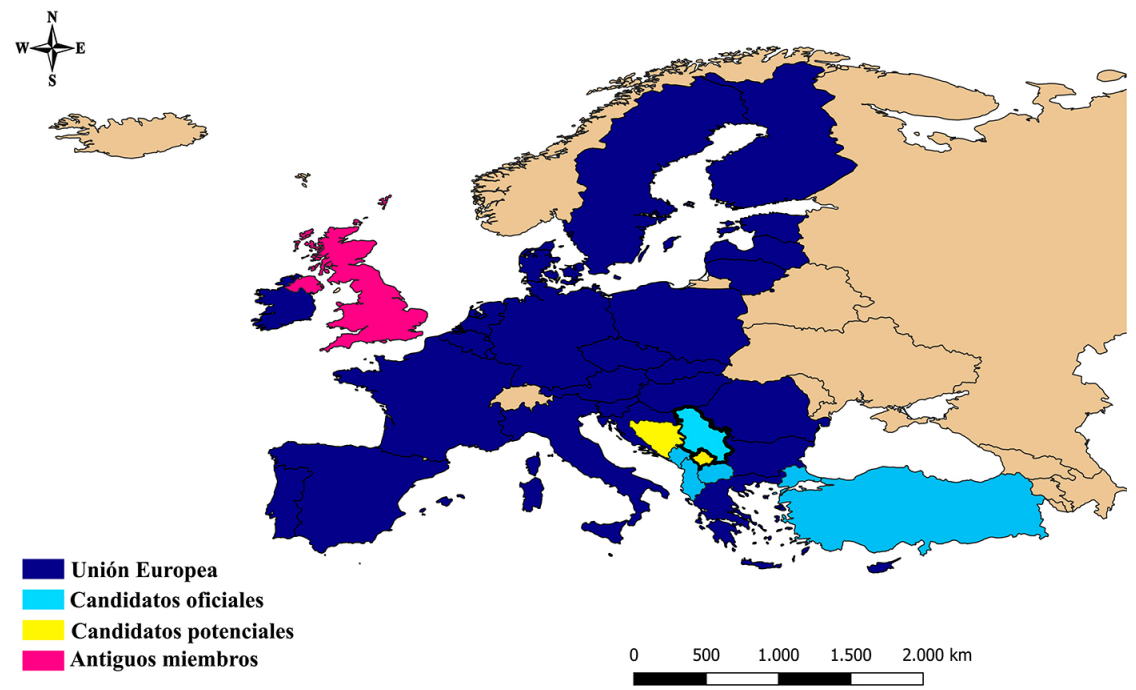

Fuente: elaboración propia.

y sobre el que Serbia sigue reclamando su soberanía, caracterizado por unas relaciones interétnicas antagonistas y con escasa cooperación (Krasniqi, 2012).

La propuesta de intercambio de territorios entre Serbia y Kosovo para hacerlos coincidir con líneas étnicas es debatida en ambos países, así como en la Unión Europea, los Estados Unidos y Rusia. Giband y Vicente (2018: 425) escriben que "comprender las fronteras de hoy en día es interpretar los procesos sociales que alimentan dos dinámicas opuestas: desfronterización y refronterización». Este proceso de refronterización serbokosovar (diferenciación, construcción de la identidad), paradójicamente, tiene en sus respectivos gobiernos la prioridad de integración en la UE, que más bien fomenta una desfronterización (obertura, intercambio, hibridación). Serbia es candidato oficial de ingreso desde 2012, mientras que Kosovo es considerado un candidato potencial (mapa 1), todavía no reconocido por cinco miembros europeos, entre ellos España.

La llegada de Donald Trump a la presidencia estadounidense fue vista con inquietud respecto a su política exterior y con la conjetura de buscar algún acuerdo internacional para presentarlo como un éxito. La controvertida decisión de reconocer los altos del Golán a Israel y la declaración del secretario Mike Pompeo sobre que «su decisión era simplemente reconocer los hechos en el terreno y la realidad» (National Review, 28/03/2019) pusieron de manifiesto que la idea de la corrección de la frontera serbokosovar podría realizarse, más aún teniendo en cuenta que el consejero de Seguridad Nacional de los 
EE. UU., John Bolton, se había mostrado partidario de esta propuesta (ESI, 2019). Ante una UE múltiplemente dividida sobre el caso kosovar (su independencia, relaciones y propuesta de intercambios), la ausencia de declaraciones alentó la propuesta y reflejó la ineficacia de la UE en los Balcanes, hasta un reciente anuncio en el que se afirmaba que tal intercambio no se encontraba en la agenda (Europa Press, 14/05/2020). La reciente cumbre prevista para el 27 de junio de 2020 en Washington entre Vučić ${ }^{1}$ y Thaçi ${ }^{2}$, auspiciada por el enviado especial para las negociaciones de paz Grennell, parecía augurar que el acuerdo era inminente, si bien fracasó. Mientras tanto, la sociedad kosovar se encuentra dividida ante esta propuesta (Kosovo 2.0, 17/02/2019).

Este artículo tiene como objetivo analizar la propuesta de intercambio de territorios (land swap) para apuntar sus implicaciones positivas y negativas, desde la teoría de las correcciones de fronteras y una perspectiva multidisciplinar de geografía política, derecho internacional público y seguridad internacional. La hipótesis es que, paradójicamente, un acuerdo consensuado podría exacerbar movimientos irredentistas y provocar conflictos en una región con una amplia dispersión de minorías étnicas. Se ha consultado una extensa bibliografía y una amplia selección de notas de prensa, y se ha visitado dichos países. El artículo se estructura en un primer apartado sobre la teoría de las correcciones de fronteras y sigue con una descripción geográfica del área de estudio. Continúa con una revisión del contexto histórico desde la creación del mito de Kosovo, seña de identidad serbia, hasta la desintegración yugoslava y la declaración de independencia kosovar. Posteriormente, se describe la situación actual en el norte de Kosovo y se analiza la propuesta de intercambio de territorios, para finalizar con la discusión de la hipótesis y las conclusiones.

\subsection{Marco teórico}

Las fronteras comportan el objeto de estudio de diferentes disciplinas, como la geografía política, el derecho internacional público y los estudios estratégicos y de seguridad internacional. Entre los geógrafos, destacan la perspectiva ratzeliana del «espacio vital», que las define como el soporte del crecimiento (Ratzel, 1896), y la posibilista, en la que frontera es vista como un marco provisional o permanente, donde son los grupos humanos los que delimitan el territorio según sus "géneros de vida», y que debe estudiarse con relación a los grupos que separa (Ancel, 2016 [orig. 1936]: 132). Las fronteras, que separan de «lo otro", varían con el tiempo, y sus cambios han sido constantes en la historia. Frecuentemente han intentado aglutinar a un mismo pueblo en sus límites,

1. Aleksandar Vučić es presidente de Serbia desde 2017. Anteriormente fue primer ministro de Serbia desde 2014.

2. Hashim Thaçi es presidente de Kosovo desde 2016. Anteriormente fue primer ministro de Kosovo cuando declaró su independencia unilateral en 2008. También fue fundador del Ejército de Liberación de Kosovo (UÇK). 
aunque ello supusiera trasvases de población o la creación de corredores, como en el caso del «corredor polaco» (Hartshorne, 1937).

El surgimiento de los Estados nación tuvo su momento álgido en el siglo XIX, con el auge del romanticismo y los ejemplos de movimientos de unificación alemán e italiano o la descolonización, que buscaban identificar a cada Estado con una sola nación. Kymlicka y Straehle (2001: 62) argumentan que la formación de los Estados nación es un proceso y no un hecho consumado, pues «son producto de deliberadas políticas de construcción nacional, adoptadas por los Estados para difundir y fortalecer un sentido de pertenencia nacional». Desde esta perspectiva, las fronteras de un mismo pueblo o nación deben coincidir con las estatales, es decir, su jurisdicción. Como señala Cairo (2001: 34), «el trazado de líneas fronterizas forma una parte fundamental de la construcción del Estado nación moderno, no se puede entender el mismo sin la conformación de un espacio homogéneo y perfectamente delimitado", además de que el uso de la territorialidad y la identificación de los habitantes con el Estado produce una legitimación de las guerras, "que se convierten en guerras populares, so pretexto de defensa del territorio nacional» (Cairo, 2001: 32).

La organización de los Estados es diversa y encontramos ejemplos plurinacionales (más de una nación en un mismo Estado) o naciones sin Estado, repartidas en diferentes países (como el caso kurdo). De igual manera, en numerosos casos la delimitación de las fronteras ha sido realizada por las potencias colonizadoras, sin tener en cuenta la distribución de los grupos étnicos, de modo que se han creado «fronteras artificiales». Estas han ocasionado conflictos que aún perduran en el tiempo: refiérase al reparto de África en la Conferencia de Berlín (1885) y a la independencia de estos territorios en el proceso de descolonización, que originó conflictos entre Somalia y Etiopía; Eritrea y Etiopía; Nigeria y Camerún; o Sudán, por nombrar algunos. Posteriormente, en el siglo XX ha ido ganando peso la formación de entidades supranacionales (políticas, económicas, militares), que suponen la dirección opuesta al proceso de determinación, ya que su pertenencia obliga a ceder ciertos aspectos de la soberanía (Velázquez y Pérez, 2010), por lo que algunos autores sostienen que nos encontramos ante el fin del «Estado nación» (Guéhenno, 2000). La disolución de la URSS y del bloque comunista condujo a la transición al capitalismo y al surgimiento de movimientos nacionalistas que buscaban consolidar su propio Estado (Martínez i Edo, 1993). Además, no hay que olvidar que el proceso de globalización amplifica los movimientos y extiende sus motivaciones y exigencias de una igual consideración en contextos geográficos bien diferenciados, por lo que decisiones singulares pueden ser vistas como un precedente en otros ámbitos más lejanos. En el contexto de la ampliación europea, resulta contradictorio aplicar a la vez estos procesos de desfronterización y refronterización (Giband y Vicente, 2018), principalmente en el Este de Europa, donde se ha variado desde las anteriores fronteras nacionales semipermeables a una frontera brusca que representa el límite exterior de la Unión Europea (Bürkner, 2020: 547). 


\subsection{Las correcciones de fronteras}

Tras la Segunda Guerra Mundial, el régimen internacional de fronteras se ha basado en el principio de integridad territorial de los Estados soberanos y en el statu quo de las fronteras existentes, independientemente de su inconsistencia (Zacher, 2001). Con la desintegración de la URSS y Yugoslavia, se asumió que las entidades de un Estado federal pueden ser reconocidas como Estados independientes en sus límites administrativos previos si cumplen con ciertas condiciones de estatalidad (statehood). En este momento, el derecho internacional público y los estudios estratégicos y de seguridad internacional alcanzan un papel relevante en cuanto a los cambios de fronteras. Al efectuar reclamos territoriales, los derechos históricos son comúnmente apelados: tanto el de la primera ocupación como el de cesiones ilícitas o ilegales (Murphy, 1990). Por ejemplo, en el caso de Crimea se resaltan tres teorías interrelacionadas: autodeterminación nacional, necesidades estratégicas basadas en geopolítica y derechos históricos (Forsberg y Mäkinen, 2019: 214). Hopf (2016) explica cómo la evolución del discurso sobre la identidad rusa hizo posible que la anexión de Crimea y la intervención militar rusa fueran vistas como naturales. Aunque internacionalmente fue considerada una anexión, técnicamente fue una secesión y una posterior integración en la Federación Rusa. El reconocimiento ruso de esta modificación de las fronteras constituyó un punto de inflexión tanto en las relaciones exteriores rusas como en su propia política de fronteras. Además, dio lugar a la independencia autoproclamada de las Repúblicas Populares de Donetsk y Lugansk (RPD y RPL) y a conjeturas sobre la visibilización de fronteras que permanecen ocultas en espacios disputados (Bernabé-Crespo, 2020). Estas fronteras interiores son especialmente importantes donde los derechos civiles son definidos con relación a las comunidades étnicas (Dahlman, 2017).

Esto enlaza con la secesión y la independencia unilateral, ya que se crean fronteras o se elevan de categoría. Moore (1998) destacó que existen dos consideraciones respecto de la autodeterminación como justificación para la secesión: un derecho irremediable cuando la población es oprimida por el Estado patrón o cuando sea vista como "positiva» incluso en ausencia de opresión política. Waters (2020: 9) identifica cuatro formas de justificar una secesión: como propiedad, nacionalismo, justicia y elección democrática. Además, estos se superponen: los nacionalistas afirman un derecho inherente, pero a menudo también creen que fueron los ocupantes originales y que han sufrido injustamente, y si son mayoría, también reclaman una base democrática. En el caso kosovar, se enfatizó que las minorías tienen el derecho de autodeterminación si sus derechos son suprimidos por el Estado, por lo que el reconocimiento podría ser visto como una vía para estabilizar la situación política (Forsberg y Mäkinen, 2019, 215). Este argumento es el que esgrime Waters (2020), en el que añade que cualquier argumento en pro de la secesión debe buscar un cese de la hostilidad.

Autores como Simmons (2005) u Oswiak y Rider (2013) estiman que los acuerdos fronterizos pueden reducir la rivalidad interestatal y promocionar la 
interdependencia económica. Para corregir fronteras existen tres mecanismos: el intercambio de territorios, la creación de corredores o la frontera permeable y abierta, como en el caso de Baarle-Hertog y Baarle-Nassau (Bélgica y Países Bajos). Ejemplos de correcciones de fronteras se han probado exitosos en diferentes áreas: en Bangladés e India, o entre Kirguistán y Uzbekistán, una frontera que desde 1999 vivió periodos de hostilidad y despliego de tropas. En 2001, ambos primeros ministros firmaron un acuerdo para establecer corredores y conectar los enclaves de Sokh a Uzbekistán y de Barak a Kirguistán, que no llegó a materializarse por el desacuerdo sobre la calidad de los territorios cedidos. Finalmente, en 2017 se firmó en Bishkek un acuerdo para delimitar el $85 \%$ de la frontera común y acordar la gestión de dos embalses transfronterizos (Toktogulov, 2018: 101), aunque todavía queda el 15\% restante y otro embalse.

Otros expertos como Dahlman y Ó Tuathail (2005) o Jeffrey (2007) creen que la multiplicación de nuevas fronteras y arreglos territoriales que dividen poblaciones étnico-nacionales puede socavar procesos largos de paz. La modificación de los límites interiores también comporta riesgos y produce debate. En Estados plurinacionales, el modelo de poder compartido (power-sharing model) implica la participación y la representación de los principales grupos étnicos en las instituciones, lo que puede ser visto como una herramienta útil para la gestión inicial posconflicto, más que una estrategia a largo plazo (Baliqi, 2018). Este fue el modelo introducido en Kosovo por las Naciones Unidas en 2001 (Bieber y Keil, 2009). La descentralización ha sido ampliamente extendida como una táctica diplomática para acabar con conflictos armados al permitir tanto la integridad territorial como una mayor autonomía de las minorías (Rothchild y Lake, 2005: 120). Lijphart (1977) introdujo el término consociacionalismo, el cual puede implicar prácticas como la autonomía territorial, acuerdos federales o confederales, federaciones basadas en poblaciones étnicas, representaciones proporcionales en la administración, o sistemas electorales proporcionales. En definitiva, enfatiza la cooperación entre las élites multiétnicas y desarrolla una cultura política del consenso, lo que se puede traducir en una reducción del conflicto potencial en sociedades divididas. Horowitz (1993), Aitken (2007) y Finlay (2011) se muestran críticos con el consociacionalismo, ya que estos acuerdos a la larga no presentarían beneficios al institucionalizar identidades antagonistas y sus divisiones, lo que impide la reconciliación.

\section{3. Área de estudio}

Kosovo es un Estado parcialmente reconocido situado en el sureste de Europa, en la península balcánica, de $10.877 \mathrm{~km}^{2}$. Un territorio montañoso y sin acceso al mar, dividido en tres vertientes hidrográficas hacia el Danubio (mar Negro), Egeo y Adriático. La economía de Kosovo es precaria y depende en gran medida de la agricultura y de la industria minera. Las minas de Trepča son las más relevantes, con depósitos de carbón, zinc, plata y otros minerales (KASA, 2013). Su población se estima en 2.200 .000 habitantes y es la más joven de 
Tabla 1. Porcentaje de población albanesa y serbia en Kosovo según los últimos tres censos

\begin{tabular}{lrrr}
\hline Grupos étnicos & 1981 & 1991 & 2011 \\
\hline Albaneses & 77,4 & 81,6 & 92,9 \\
Serbios & 13,2 & 9,9 & 1,5 \\
Otros & 9,4 & 8,5 & 5,6 \\
\hline
\end{tabular}

Fuente: elaboración propia a partir de Djukanović (2008) y ASK (2012).

Europa (KASA, 2013: 9), la cual, según el censo de 2011, es de mayoría albanesa $(92,9 \%)$. Siete grupos étnicos son reconocidos como minorías oficiales: serbios $(1,5 \%)$, bosníacos $3(1,6 \%)$, turcos $(1,1 \%)$, ashkali $(0,9 \%)$, egipcios $(0,7 \%)$, gorani $(0,6 \%)$ y roma $(0,5 \%)$. Los albaneses profesan en su mayoría la religión musulmana (bektashi, rufai, saadi, melani, nakshbandi) y una pequeña parte de ellos son católicos. Los serbios son cristianos ortodoxos y los turcos son musulmanes. El grupo de roma-ashkali-egipcios es más heterogéneo, ya que la mayoría son musulmanes, pero también hay ortodoxos. Los gorani son eslavos con un lenguaje característico, pero profesan la religión musulmana y se concentran sobre todo en Brod y Restelica, al sur de Prizren (KASA, 2013). Como señala Demjaha (2016), la relación entre albaneses y serbios es la que causa más tensiones, mientras que los grupos restantes se han integrado mejor en el país. La tabla 1 muestra la evolución de la población serbia y albanesa en Kosovo, aunque es de destacar que el censo de 1991 fue boicoteado por los albaneses y el de 2011 por los serbios (y no incluye a los municipios del norte de Kosovo: Leposavić, Zubin Potok, Zvečan y Mitrovica norte). Esta parte norte de Kosovo ${ }^{4}$ se encuentra bajo control serbio, aunque es reclamada por el Gobierno kosovar como una parte integral de la república, ya que este declaró su soberanía sobre el territorio que constituía la anterior división provincial.

Serbia continúa considerando a Kosovo como una de sus provincias autónomas, al igual que Voivodina (mapa 2). Por su parte, el Valle de Preševo es un área de $725 \mathrm{~km}^{2}$ situada en el sur de Serbia, limítrofe con Kosovo al oeste, y es una denominación geográfica y cultural, pero no constituye una unidad administrativa. Este es un valle drenado por el río Moravica y el Morava occidental, que representa la comunicación natural de Serbia con Macedonia del Norte y el mar Egeo. Compuesto por tres municipios (Preševo, Bujanovac y Medveđa), la población albanesa ha rechazado participar en los censos serbios desde 2011 (Lasserre, 2019: 25), aunque Islami y Ejupi (2015) estiman que el $80 \%$ está formado por albanokosovares.

3. Bosníaco es el nombre que se le da a la etnia bosnia que profesa la religión musulmana (y que se encuentra principalmente en Bosnia y Herzegovina y en otros territorios como el Sandžak); no confundir con el gentilicio bosnio, que hace referencia al conjunto de ciudadanos del país de Bosnia y Herzegovina.

4. Sobre la diferenciación entre «norte de Kosovo» y «Kosovo del Norte»: la primera denominación es una referencia meramente geográfica (y preferida por la postura albanokosovar); la segunda implica una entidad propia administrativa, la cual no existe de iure pero sí de facto. 
Mapa 2. Área de estudio
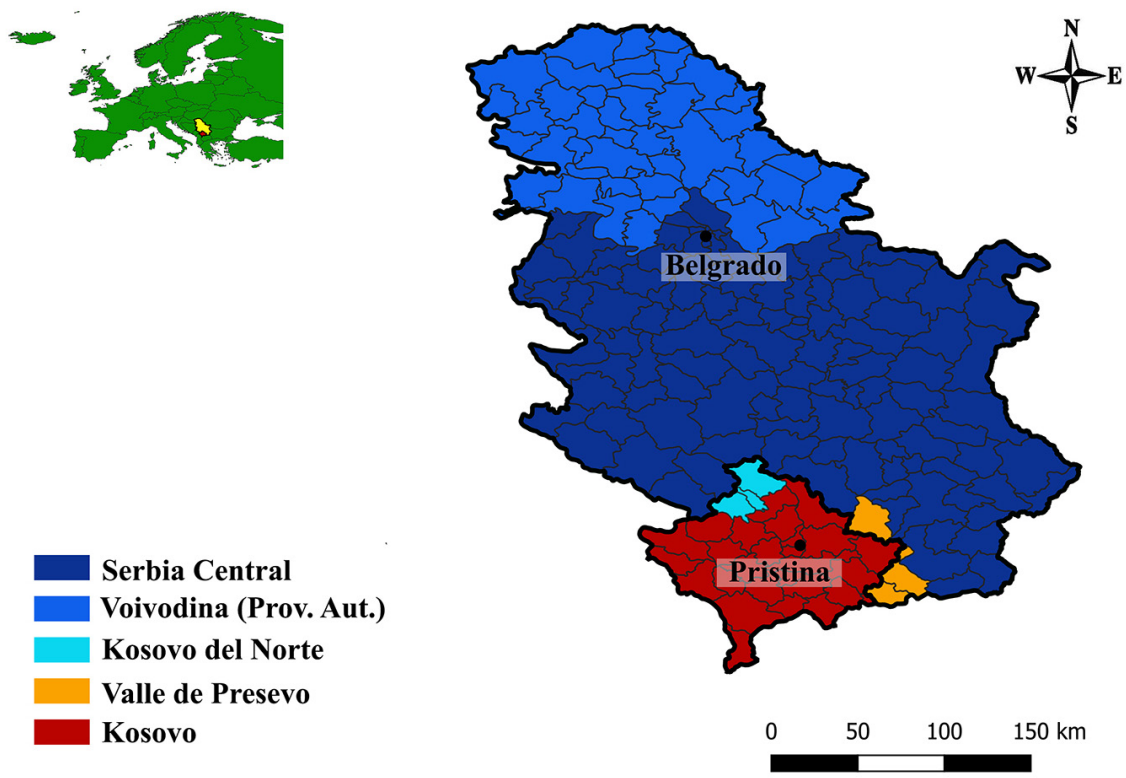

Fuente: elaboración propia.

2. Contexto histórico: del mito de Kosovo a la declaración de independencia y el diálogo de Bruselas

\subsection{La creación del mito de Kosovo}

A menudo referido como «la cuna de la nación serbia», el mito de Kosovo tiene su origen en la batalla del campo de los mirlos o Batalla de Kosovo, en 1389, cuando los serbios cayeron derrotados ante el avance otomano, y su territorio, lleno de monasterios (como el de Gračanica - figura 1-, el de Dečani o el Patriarcado de Peć), quedaba fuera de sus manos. Malcolm (1998) argumenta que albaneses y serbios vivían en el mismo territorio desde hacía siglos, salvo que los primeros habitaban en las zonas más montañosas y los segundos en los valles, por lo que la relación era escasa. De hecho, en el siglo III dC ya se constata la existencia del reino de Dardania ${ }^{5}$ por tribus ilirias. Además, el territorio conocido como la Ras de Serbia, germen del Estado serbio, se encontraba más al norte, en el territorio que hoy ocupa Novi Pazar y Raška.

El dominio otomano estableció en el siglo XIX el vilayeto de Kosovo. Tras siglos de dominio otomano, en el XIX se confirmó la independencia serbia, y posteriormente se conquistó el área de Kosovo (llamado por los serbios Kosovo i Metohija, esta última palabra quiere decir 'tierras de la iglesia' o 'tierras

5. El nombre de Dardania parece estar vinculado a la palabra albanesa dardhë (pera). 
Figura 1. Monasterio de Gračanica, Kosovo

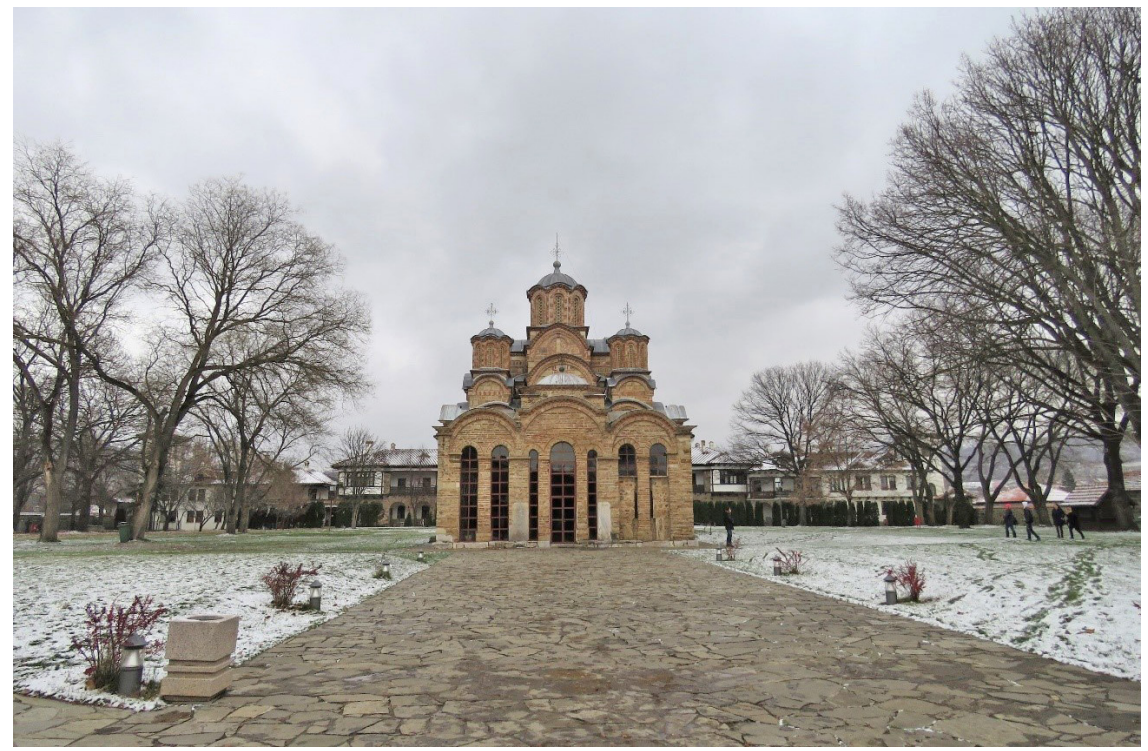

Fuente: autor, 29/11/2016.

Figura 2. Liga de Prizren, Kosovo

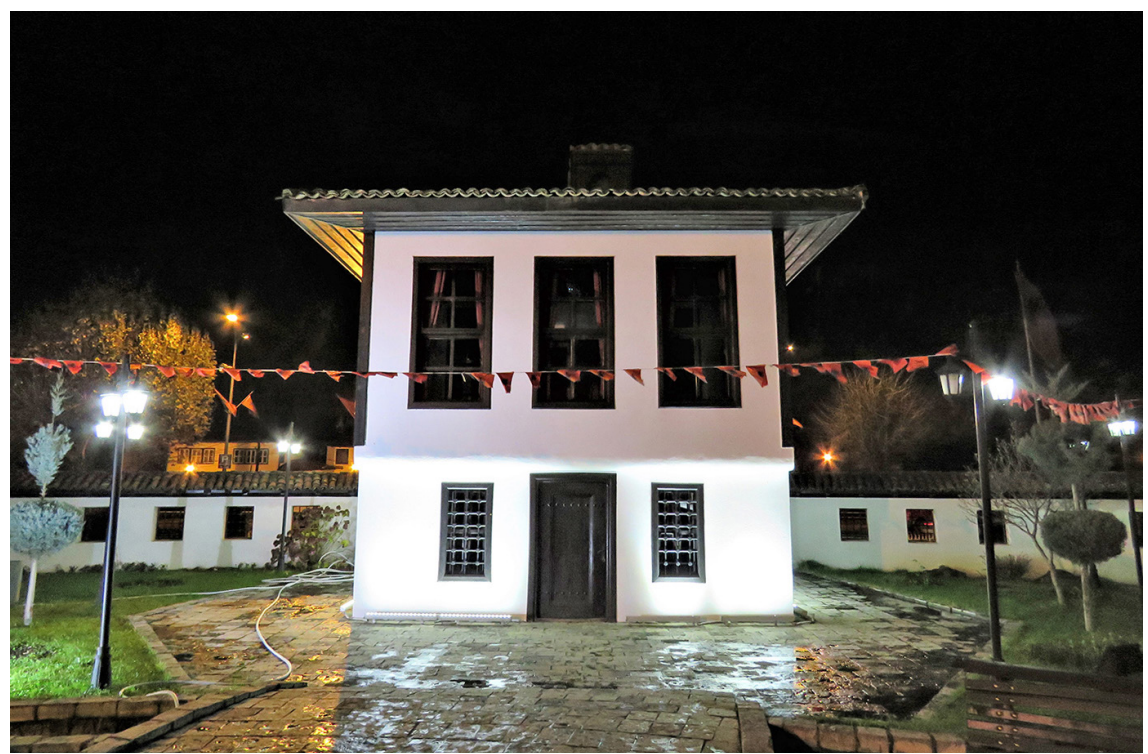

Fuente: autor, 28/11/2016. 
monásticas'), y se asistió al proceso de creación del mito de Kosovo, que, aunque había pervivido en la tradición oral, ahora era ensalzado y símbolo de identidad (Marković et al., 2019). En 1878 se formaba en Kosovo la llamada Liga de Prizren (figura 2), una organización para la defensa de los derechos de los albaneses que perseguía el establecimiento de un Estado albanés. De esta manera, Prizren es también visto por la población albanesa con especial atención como el germen de su Estado (KASA, 2013).

\subsection{Desde la Primera Guerra Mundial hasta la disolución de Yugoslavia}

Al fin de la Primera Guerra Mundial, se conformó el Reino de los Serbios, Croatas y Eslovenos, en 1929 renombrado como Reino de Yugoslavia. Tras la Segunda Guerra Mundial, se estableció sobre la República Federal Socialista de Yugoslavia (RFSY), cuya constitución incluía la Provincia Autónoma de Kosovo y Metohija dentro de la República Socialista de Serbia. En 1968 hubo protestas estudiantiles en Pristina para elevar el rango al de república, a la misma vez que cambiar el nombre de Kosovo y Metohija al de Kosovo y Dukagjin ${ }^{6}$ (KASA, 2013: 15), algo que se consiguió en parte, pues la Constitución de 1974 de la RFSY dio una mayor autonomía a la renombrada Provincia Socialista Autónoma de Kosovo. Sin embargo, tras la muerte de Tito y el ascenso del nacionalismo serbio con Milosevic, la autonomía fue suspendida (Kitanics y Pap, 2012). En 1989, el $600^{\circ}$ aniversario de la Batalla de Kosovo y el discurso de Milosevic en Mitrovica contra los albanokosovares reavivaron dicho mito en un contexto de auge nacionalista en las repúblicas. Se produjo el detonante para las guerras yugoslavas: Eslovenia y Croacia fueron las primeras en declarar su independencia, seguidas de Macedonia y Bosnia y Herzegovina. Kosovo también declaró su independencia mediante un referéndum — pero no recibió atención ni reconocimiento internacional (Bieber, 2015)—, en el que casi el $100 \%$ de los albanokosovares apoyaron la idea de un Estado independiente (Calić, 2000: 22) e incluso llegó a aprobarse una Constitución en Kaçanik.

La República Federal de Yugoslavia (RFY) fue la sucesora de la RFSY, y se lanzó una nueva ofensiva contra la población albanesa en Kosovo en 1998 que comportó más episodios de limpieza étnica ${ }^{7}$. En 1999 se produjo la intervención de la OTAN mediante el bombardeo a posiciones serbias, y la Resolución 1244 del Consejo de Seguridad de las Naciones Unidas puso a Kosovo bajo un protectorado internacional mediante la UNMIK (United Nations Interim Administration Mission), que gradualmente fue construyendo instituciones autónomas. Este mismo año, el Plan de Rambouillet proponía amplios derechos para las comunidades nacionales para organizar sus propias instituciones democráticas y administrar sus asuntos (Dahlman, 2017), aunque fue recha-

6. Dukagjin es el nombre albanés para referirse a las tierras altas del oeste de Kosovo, donde se encuentran Peć/Peja, Đakovica/Gjakova y Prizren.

7. En los años 80 comenzaba a emplearse el término «limpieza étnica» entre los activistas serbokosovares, preocupados por el «factor étnico», que describía a Kosovo como vaciado de serbios (Vladisavljević, 2004: 786). 
zado por Serbia y Rusia. A pesar de su fracaso, las negociaciones supusieron tres mecanismos para el futuro: restablecer el municipio como escala para las negociaciones, que estas serían tomadas en base a los intereses étnicos mayoritarios, y que las negociaciones contemplarían una asociación de municipios para formar una entidad territorial inespecífica.

La propuesta de Martti Ahtisaari, anterior presidente de Finlandia y entonces enviado especial de la ONU en Kosovo, de marzo de 2007, llamada Propuesta Integral para el Acuerdo sobre el Estado de Kosovo (Comprehensive Proposal for the Kosovo Status Settlement), reiteraba que la independencia era la única solución viable y recogía la limitada capacidad de Kosovo para garantizar la protección de las minorías, de desarrollar instituciones democráticas, así como de desarrollar su economía. También estipulaba que debía contar con la supervisión y el apoyo de autoridades internacionales civiles y militares. Esta propuesta fue fuertemente rechazada por Serbia y Rusia — esta última amenazó con vetar cualquier resolución en el Consejo de Seguridad de la ONU (Demjaha, 2016: 186).

\subsection{La declaración de independencia de Kosovo y el diálogo de Bruselas}

El 17 de febrero de 2008, el Parlamento de Kosovo promulgó la declaración de independencia «de plena conformidad con las recomendaciones del enviado especial de las Naciones Unidas Martti Ahrisaari», y se declaró a Kosovo como una república democrática, secular y multiétnica, y que aceptaba totalmente las obligaciones recogidas en la propuesta de Ahtisaari. La esfera occidental aceptó la declaración unilateral de independencia (Ker-Lindsay, 2009; Bieber, 2015), aunque no fue reconocida por Rusia y otros países, entre ellos España y otros de la UE (Grecia, Chipre, Rumanía y Eslovaquia).

La controvertida aceptación de la independencia dividió y sigue dividiendo al mundo: actualmente, 97 o 98 países reconocen su independencia, número difícil de cuantificar debido a la variedad de interpretaciones sobre el reconocimiento (Ker-Lindsay, 2012). Condolezza Rice, secretaria de Estado de los EE. UU., reiteró en numerosas ocasiones la combinación de factores que apoyaban esta decisión: el contexto de la desintegración yugoslava, la historia de limpiezas étnicas y los crímenes contra civiles en Kosovo, y que el periodo prolongado de la administración bajo las Naciones Unidas no se encontraba en otro lugar salvo en este caso aislado y que no podía constituir un precedente. Esta fue una excepción sui generis a la práctica existente más que una nueva práctica (Fabry, 2012: 666). Posteriormente, en julio de 2010, la Corte Internacional de Justicia concluyó que la declaración de independencia del 17 de febrero de 2008 no violó la ley general internacional (ICJ, 2010).

Ante una UE dividida sobre el estatus de Kosovo, en septiembre de 2010 se apostó por una apertura al diálogo materializada en la Resolución 64/29 por la Asamblea General de la ONU, en la que se atribuye a la UE la responsabilidad de facilitar el proceso de diálogo entre Pristina y Belgrado. Solo después de que 
se hubiera abierto, el 8 de marzo de 2011, Serbia ganó el estatus de candidato oficial a la membresía europea el 2 de marzo de 2012.

Este diálogo Pristina-Belgrado, referido como «normalización de relaciones» y mediado por la UE, ha sido una prioridad en la agenda europea, y uno de sus logros fue el «Primer Acuerdo de Principios que rige la Normalización de Relaciones» de 2013. En él, Serbia propuso algo que ya se recogía en el Plan de Rambouillet: la creación de una Comunidad/Asociación Autónoma de los Municipios Serbios (Zajednica Srpskih Opština, ZSO), de la que formarían parte los cuatro situados en el norte de Kosovo y otros seis de mayoría serbia del resto del territorio kosovar, en los que tendrían competencias en economía, educación, sanidad y planeamiento urbano y rural (Demjaha, 2016: 191). En 2015 se finalizó el acuerdo con las adiciones relativas a la energía, telecomunicaciones y la libertad de movimiento en el puente de Mitrovica, aunque sin haberse llegado a su total implementación.

\section{La situación actual en Kosovo del Norte y Valle de Preševo}

Demjaha (2016: 182) escribía que las relaciones interétnicas son asimilables a las relaciones entre Estados, por lo que el trato de las minorías adquiere una importancia añadida y de relación con el vecindario. La Constitución de la República de Kosovo (capítulo I, artículo 3) define al país como «una sociedad multiétnica que consiste en albaneses y otras comunidades». La protección de los derechos de las minorías está recogida en el artículo 57.1, capítulo III de la Constitución de Kosovo, sobre los Derechos de las Comunidades y sus Miembros. Por ejemplo, el serbio es idioma oficial en todo Kosovo, inclusive en áreas donde los serbios no son mayoría.

Según Baliqi (2018: 58), el actual sistema electoral de Kosovo refuerza la división étnica al institucionalizar las diferencias políticas entre las comunidades, ya que los representantes son elegidos solo dentro de las comunidades étnicas. De esta manera, se reservan 20 de los 120 asientos en el Parlamento para la representación de las minorías, de las cuales la serbia tiene garantizados 10 como mínimo (Constitución de la República de Kosovo, 2008: 57). Además, cualquier modificación de la Constitución de Kosovo necesita la aprobación de dos tercios del Parlamento, con la inclusión de dos tercios de los miembros de los representantes de las minorías (artículo 144, Constitución de la República de Kosovo). En consecuencia, normalmente se suele prestar poca atención a los temas económicos y sociales, ya que en gran parte el voto está condicionado a la etnia. De esta manera, Calu (2020) concluye que la implementación de los derechos de las minorías ha creado una jerarquía entre los diferentes grupos étnicos, lo que puede ser contraproducente y causar segregación en vez de integración.

\subsection{Kosovo del Norte}

Kosovo del Norte, compuesto por los municipios de Leposavić, Zubin Potok, Zvečan y Mitrovica norte, es un territorio controlado de facto por Serbia, y 
constituye enclaves étnicos, lingüísticos, religiosos y de simbología nacional. En esta área existen instituciones paralelas establecidas y apoyadas por Belgrado (Borgh, 2012). Esto se traduce en que la capacidad y voluntad de abordar una integración en las instituciones de Kosovo es dictada según la política serbia (Matveeva y Paes, 2003). De igual manera, la falta de autoridad central durante dos décadas ha propiciado una élite de políticos fuertes y crimen organizado que son los que en la práctica manejan la región (Rossi, 2018b).

El norte de Kosovo es un espacio donde se siguen produciendo las mayores tensiones entre las dos comunidades étnicas, con esporádicos brotes de violencia, algo que es complementado con declaraciones como la que el presidente serbio Vučić hizo en televisión acerca del conflicto de Kosovo: «Están esperando el momento para atacar a los serbios en el norte de Kosovo» (RTS, 02/03/2019).

La situación del resto de serbios en Kosovo es diferente y varía según los municipios, ya que su distinta integración ha creado múltiples identidades serbokosovares dentro del territorio de Kosovo (Fort, 2018). Aunque se vive una calma tensa, el conflicto continúa latente. Por ejemplo, el 17 de marzo de 2004 se produjeron disturbios violentos contra los serbios y su legado patrimonial a lo largo de Kosovo, el episodio más violento desde el final de la guerra, que tuvo como resultado 19 muertos, más de 900 heridos, 4.500 personas desplazadas y más de 700 casas de serbios, ashkali y roma dañadas (Demjaha, 2016, 186).

\subsection{El Valle de Preševo}

Según Huszka (2007), los albaneses del Valle de Preševo tienen más dificultades para trabajar en el sector público serbio y su tasa de desempleo es mayor. Zejnullahi (2015: 92) también relata carencias en el sistema sanitario y en el educativo, y de uso de la lengua albanesa o empleo de símbolos nacionales. Su situación y lazos con el resto de albanokosovares motivó que se estableciera entre 2000 y 2001 el Ejército de Liberación de Preševo, Medveđa y Bujanovac (UÇPMB, en albanés), formado por antiguos miembros de la UÇK (Ejército de Liberación de Kosovo), disueltos por los acuerdos de Konculj y la implementación del Plan Cović, cuyo propósito era mejorar la integración de los albaneses de Preševo en Serbia (Bernabé-Crespo y Peña-Ramos, 2019). Sin embargo, Pristina se ha referido alguna vez al Valle de Preševo como «Kosovo del este» (Rossi, 2018b), y el mismo alcalde de Bujanovac expresó en 2017 que la Gran Albania debería incluir el Valle de Preševo e incluso llegar hasta la ciudad serbia de Niš (b92, 21/04/2017).

\subsection{El Diálogo de Bruselas con relación al norte de Kosovo}

De las negociaciones realizadas en el Diálogo de Bruselas, sobresale la falta de referencias directas hacia el futuro de los serbokosovares (Gashi et al., 2017: 544), por lo que muchos sienten que no están representados en el diálogo ni 
Figura 3. Estatua de Miloš Obilić ${ }^{\star}$ con la bandera serbia en Gračanica (Kosovo)

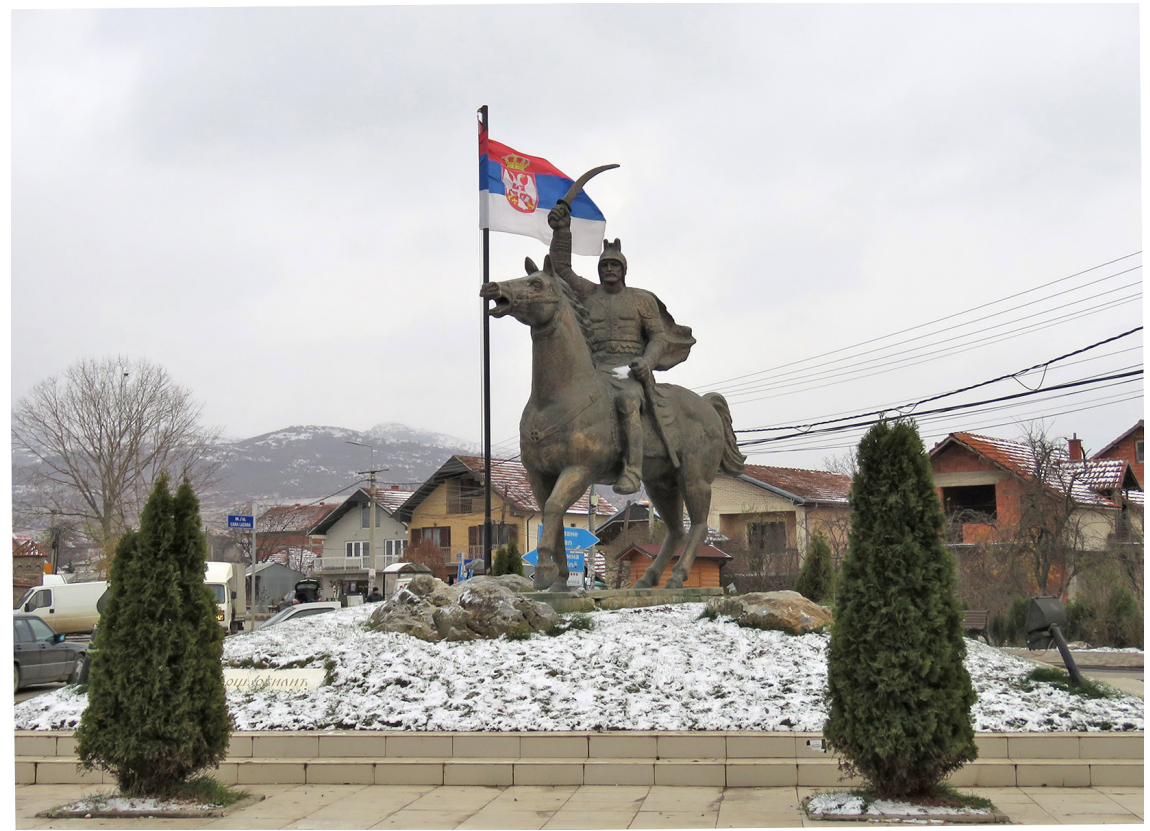

* Miloš Obilić es un héroe nacional serbio que luchó en la Batalla de Kosovo de 1389 y asesinó al sultán otomano.

Fuente: autor, 29/11/2016.

por la parte kosovar ni por la serbia. Dahlman (2017) también critica que dichos acuerdos son una manera de gestionar la competición nacional a través de la segregación local. El acuerdo de 2015 no llegó a implementarse del todo debido a las críticas de los partidos de la oposición, que temían una federalización del país y una interferencia de Serbia en los asuntos internos de Kosovo (Demjaha, 2016: 192). De igual manera, la descentralización también ha redibujado límites municipales internos en Kosovo, con la creación de nuevos municipios de mayoría serbia (Mitrovica del norte, Gračanica - figura 3-, Ranilug, Partes, Klokot y Novobrdo) y uno de mayoría turca (Mamusha), según la Ley de Autonomía Local no 03/L-040 (Baliqi, 2018: 60).

El proceso de diálogo se encuadra dentro del contexto de integración europea, lo que al ser una prioridad para ambos países coloca a la UE en una fuerte posición, aunque la falta de unidad en la UE respecto del estatus de Kosovo socava tal autoridad. De hecho, el Acuerdo de Estabilización y Asociación (SAA, en inglés) de Kosovo y el estatus de candidato oficial de Serbia estuvieron directamente condicionados por el avance en esta normalización de relaciones. Entre otros, la membresía de Kosovo en la UE confirmaría su reconocimiento y estatalidad, por lo que el proyecto europeo cuenta con una gran 
popularidad en la sociedad (Gashi et al., 2017: 540). Vučić, por su parte, es consciente de que, sin resolver el problema de Kosovo, Serbia no podrá entrar en la UE (b92, 31/05/2020). Sin embargo, el presidente serbio parece jugar en una ambigüedad intencionada, que lo posiciona tanto en posturas como que «el reconocimiento de Kosovo por parte de Serbia no está en la agenda» (b92, $11 / 06 / 2020)$, a la vez que «no descarta la posibilidad de reconocer a Kosovo (Danas, 04/03/2019).

La razón es que a Serbia se le impone un dilema para elegir entre la pertenencia al club europeo o sus pretensiones sobre Kosovo. Marković et al. (2019: 186) concluyen que, en un hipotético referéndum sobre la adhesión a la UE, el 54\% de los serbios votarían a favor, mientras que el 39\% lo haría en contra, motivados en gran parte por el asunto de Kosovo: el 79\% estima que la política europea respecto de Kosovo es influyente en su voto, y el 94\% cree que la UE apoya la independencia de Kosovo. Sin embargo, un 52\% cree que el acceso a la UE es mejor que el statu quo, y el 58\% piensa que Kosovo será un país independiente en el futuro a pesar de todo (Marković et al., 2019: 187), por lo que Serbia cuenta con poca fuerza que ejercer. Estos autores confirman que, si se confrontan estos datos y el hecho pertenecer a la UE implicara perder definitivamente Kosovo, el $63 \%$ se opondría al acceso y solo votaría a favor el 37\% (Marković et al., 2019: 189). Es por ello que desde algunos círculos se ha sugerido el intercambio de territorios como manera para solucionar el conflicto. El mismo Vučić "quiere que Serbia sea inteligente y coja lo que pueda» (RTS, 16/08/2018) y estaba «a favor de una delimitación con los albaneses en Kosovo" (b92, 09/08/2018). Por último, cabe señalar un dato significativo: el $34 \%$ de los serbios creen que la UE no tiene intención de permitir la entrada de Serbia (Marković et al., 2019: 185), lo que refleja la incredibilidad de la UE en los Balcanes, acrecentada con promesas inconclusas y dilatadas en el tiempo. Basta mencionar que todavía no se han liberalizado los visados para los kosovares, o el dilatado proceso de apertura de negociaciones de adhesión para Albania y Macedonia del Norte. Para el último caso macedonio, el acuerdo de Prespa ${ }^{8}$ probó que la resolución pacífica es posible en los Balcanes, y que el incentivo de pertenencia a la UE puede ser efectivo.

\section{Propuesta de intercambio de territorios}

\subsection{Descripción de la propuesta y motivaciones}

Como herramienta para solucionar las tensiones étnicas, con el objetivo serbio de "recuperar parte de Kosovo», el objetivo kosovar de lograr una independencia reconocida y el objetivo común de solucionar disputas que allanen

8. El Acuerdo de Prespa se firmó el 17 de junio de 2018 entre el Gobierno de Grecia (encabezado por Alexis Tsipras) y la Antigua República Yugoslava de Macedonia (con Zoran Zaev y Nikola Dimitrov al mando). El acuerdo ponía fin a la larga disputa sobre el nombre de este último país, presente desde la declaración de independencia en 1991, y que pasaba a denominarse «Macedonia del Norte». 
Mapa 3. Propuesta de intercambio de territorios entre Serbia y Kosovo

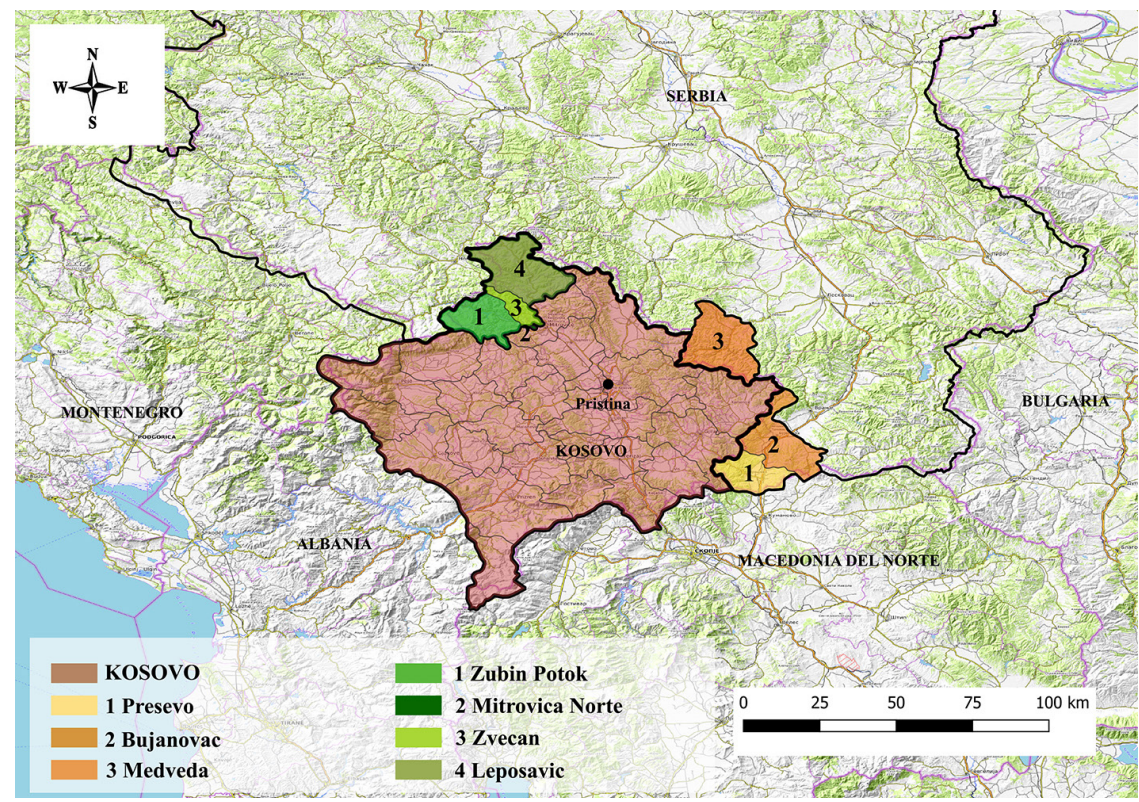

Fuente: elaboración propia.

su ingreso en la UE, la propuesta de intercambio de territorios ha cobrado fuerza desde 2018. Bieber (2017) ya señalaba que con el ascenso de Trump a la presidencia se incentivaría el énfasis de los intereses nacionales, y abría la puerta al rediseño de las fronteras y al ascenso del autoritarismo. Sin embargo, las propuestas de particiones no son algo novedoso: Spahiu (1999) ya habla de algunas que amenazaron con exacerbar el panalbanismo separatista en el sur de Serbia y norte de Macedonia. Roux (2003) recoge nueve propuestas de partición formuladas en 1991, entre las que destacaba la propuesta de 1993 del geógrafo serbio Radovanović de atribuir a Serbia el norte de Kosovo y los municipios de mayoría serbia situados al sur del río Ibar. En 2002, el viceprimer ministro serbio Cović propuso la creación de una entidad autónoma serbia en el norte de Kosovo, y anticipaba así la idea que se fraguaba de la independencia kosovar (Roux, 2003). De igual manera, una plataforma política establecida en enero de 2006 por los municipios del Valle de Preševo manifestó su deseo de unirse a Kosovo si el norte de Kosovo lo hacía con Serbia (Huszka, 2007: 7). La propuesta trata de que los cuatro municipios de mayoría serbia del norte de Kosovo (Zubin Potok, Leposavić, Zvečan y Mitrovica norte) se integren en Serbia, mientras que tres municipios de mayoría albanesa del sur de Serbia (Preševo, Bujanovac y Medveđa) se integren en Kosovo (mapa 3). Diversos medios de comunicación se han hecho eco de acuerdos concretos, como la agencia IBNA (25/02/2019), que presuntamente incluía la gestión 
conjunta de las minas de Trepča, el estatus de ciudad libre para Mitrovica, un distrito especial para Bujanovac (en el que la base militar de Jug estaría bajo administración de la OTAN), y los monasterios serbios como enclaves extraterritoriales de Serbia.

\subsection{Apoyos y potenciación de la propuesta}

La propuesta tiene como mayores valedores a diversas autoridades de ambos gobiernos. Por el lado serbio, el entonces presidente Boris Tadić apuntó ya en marzo de 2011 que «los albaneses no aceptarán ser gobernados por los serbios, y tampoco los serbios del norte por los albaneses» (Nezavisne, 27/03/2011). En 2018, cuando se reactivó la propuesta de intercambio, el ministro de Defensa, Aleksandar Vulin, explicó en febrero que la prioridad de Serbia era "parar la Gran Albania» y que "Serbia necesitaba una demarcación permanente y firme entre serbios y albaneses en el territorio de Kosovo" (Kossev, 06/02/2018). Ese mismo mes, el ministro de Asuntos Exteriores, Ivica Dačić, advertía sobre que, si los serbios eran atacados en el norte de Kosovo, tendrían que defenderlos militarmente (ESI, 2019). En mayo, Dačić reiteró que «había estado hablando sobre la partición de Kosovo los últimos diez, quince años y creía que era la única solución posible y rápida» (Blic, 28/05/2018). La presión por parte del Gobierno serbio alcanzó un máximo cuando Vučić sugirió que, si no quieren demarcación, «dentro de cuarenta años tendrán que defender Vranje ${ }^{9}$ de los albaneses» (Blic, 25/07/2018).

Del lado kosovar, el presidente Hashim Thaçi es el mayor patrocinador de la propuesta (N1, 05/02/2019). En septiembre de 2018, Thaçi expresó que «su idea era una corrección de la frontera y unificación de Preshevë, Medvegja y Bujanoci ${ }^{10}$; pero que ninguna parte de Kosovo, Gazivoda, Kosovo del Norte o Trepča serán parte de Serbia» (Kossev, 22/09/2018). El 25 de agosto de 2018, Thaçi anunció en Alpbach (Austria) que ambos presidentes estaban considerando cambios fronterizos como parte de un acuerdo para la normalización de las relaciones (ESI, 2019: 4). A ello se le suman las recientes filtraciones sobre que las instituciones kosovares habrían estado presionando para favorecer el intercambio con Serbia, entre las que se habla de $168.000 €$ de fondos públicos, desviados en 2019 desde el Gobierno del entonces presidente Haradinaj (Pristina Insight, 28/05/2020) a la agencia de comunicación francesa Majorelle PR $\&$ Events (Courrier des Balkans, 03/06/2020), al embajador francés en Kosovo y académicos estadounidenses (Pristina Insight, 09/06/2020).

El nombramiento de Bolton como Consejero de Seguridad Nacional de los EE. UU. el 9 de abril de 2018 acrecentó los rumores sobre que el acuerdo podría materializarse. El que fuera entonces embajador de los EE. UU.

9. Vranje es la ciudad más importante del sur de Serbia, a mitad de camino entre Medveđa y Preševo, pero no contemplada en el intercambio de territorios por su mayoría de población serbia.

10. Nombres albaneses para Preševo, Medveđa y Bujanovac. 
en la ONU ya había expresado que «la partición de Kosovo es lo correcto, al menos para las áreas con mayoría serbia que limitan con el sur de Serbia, ya que reflejaría la realidad étnica y política en el terreno» (Glas Amerike, 28/03/2008). En enero de 2019, el presidente de Kosovo respondió a una carta de Donald Trump en la que se relataban las intenciones de llegar a un acuerdo histórico con Serbia en la Casa Blanca (President of the Republic of Kosovo, 08/01/2019). El acuerdo parecía inminente cuando así lo expresó el presidente del Consejo Nacional de los albaneses en Serbia (b92, 27/05/2020), y tras el anuncio de que Vučić y el nuevo presidente kosovar Hoti se iban a reunir en Washington el 27 de junio de 2020 con Grennell, enviado especial de los EE. UU. para las negociaciones de paz entre Serbia y Kosovo (Joseph, 2020). Sin embargo, la reunión no llegó a finalizarse debido a la acusación de crímenes de guerra y crímenes de lesa humanidad contra Hashim Thaçi por La Haya (KSC, 24/06/2020).

\section{Discusión}

La corrección de la frontera entre Serbia y Kosovo es un asunto estratégico para ambos países y para el conjunto de la comunidad internacional, por las implicaciones que conlleva respecto del derecho internacional público y las relaciones internacionales. La implementación del ajuste de la frontera serbokosovar en los términos acordados permitiría una normalización de las relaciones, requisito fundamental de la UE para que ambos países puedan acceder al club europeo. Serbia ganaría parte del territorio de Kosovo, a sabiendas de que le es imposible recuperar el resto. Por su parte, la legitimidad de Kosovo sobre su sector norte es cuestionada, pues se identifica como Serbia, y para los norkosovares Serbia nunca ha dejado de estar presente y de funcionar como Estado allí (Rossi, 2018a). Kosovo nunca tuvo el control sobre el norte, por lo que la llamada "partición» simplemente sería el retiro del reclamo territorial sobre esta parte (Capussela, 2018). En contrapartida, Kosovo vería consolidada su independencia y su pertenencia a la ONU, ya que un acuerdo con Serbia imposibilitaría el veto ruso. En este sentido, cabe esperar que si se aceptó una declaración de independencia unilateral también debería aceptarse una decisión consensuada por ambas partes. Cabe señalar que ya se realizó una corrección de frontera exitosa entre Kosovo y Montenegro, según el acuerdo alcanzado en 2015 y ratificado en 2018 (Bakota, 2018), que significó la cesión de 8.200 ha a Montenegro y que encontró fuerte oposición en Kosovo: el principal partido de la oposición, Vetëvendosje! ${ }^{11}$, lanzó gas lacrimógeno en el Parlamento para impedir la votación (The Independent, 21/03/2018).

11. Vetëvendosje! (autodeterminación) es un partido nacionalista de izquierdas que reclama la integridad territorial de Kosovo en las fronteras establecidas internacionalmente. El partido resultó vencedor en 2019 y su presidente, Albin Kurti, se convirtió en primer ministro de Kosovo. En junio de 2020, una moción de censura de la oposición lo apartó del cargo y se eligió a Avdullah Hoti como nuevo primer ministro. 
La propuesta de intercambio de territorios tiene sus apoyos en parte de los gobiernos y asociaciones, pero también tiene una amplia gama de detractores. Las oposiciones en ambos parlamentos y gran parte de la opinión pública kosovar están en contra (Capussela, 2018). En Serbia, Marković et al. (2019: 188) señalaban que una hipotética partición de Kosovo solo sería aceptable para el $38 \%$. La Iglesia ortodoxa serbia también se opone, pues significaría perder sus monasterios. Aunque los EE. UU. se han mostrado listos para aceptar el acuerdo, parte de la comunidad internacional se ha posicionado en contra, como Alemania (Joseph, 2018). Rusia, por su parte, anunció que apoyaría cualquier decisión aprobada por el ejecutivo serbio, pero subrayó la importancia que ostenta en la región. Lavrov, ministro de Exteriores, manifestó que «la aprobación de Moscú es necesaria para cualquier acuerdo entre Serbia y Kosovo» (RFE/RL, 19/06/2020), y recordó que Rusia podía ejercer su derecho a veto en el Consejo de Seguridad de la ONU (Beta, 15/06/2020).

El acuerdo podría no ser beneficioso para los norkosovares, pues, aunque verían solucionados muchos de sus problemas, a la larga tendrían menos influencia en el conjunto político serbio (Rossi, 2018b). Serbia perdería el control sobre el estratégico Valle de Preševo, donde se encuentra la autopista que conecta Serbia con Macedonia del Norte y la vía férrea Belgrado-Skopie (y que da acceso al mar Egeo por Tesalónica). Para Kosovo significaría perder las minas de Trepča, el embalse de Gazivoda y su central eléctrica, de estratégica importancia económica para el país (Bernabé-Crespo y Peña-Ramos, 2019). Para los albaneses de Preševo, incluirse en Kosovo solo les beneficia si la soberanía de Kosovo es reconocida total e internacionalmente.

El revisionismo en los límites del Estado y los ajustes de frontera aparentan estar relacionados con la construcción de los Estados nación. Vulin, ministro de Defensa de Serbia, equiparaba la unificación alemana, «que no representó un problema para nadie», con que los «serbios deben estar unidos políticamente» (Vijesti, 26/09/2020), lo que recientemente se ha juntado con la creación del «Día de la Unidad, Libertad y Bandera Serbia», celebrado por primera vez el 15 de septiembre de 2020 (Telegraf, 15/09/2020). Además, el mayor riesgo que corre la propuesta es la posibilidad de agravar tensiones y desatar un resurgimiento de la limpieza étnica. Cabe recordar que Vodislav Šešelj, líder serbobosnio acusado de crímenes de guerra y crímenes contra la humanidad, ya sugirió en 1984 dividir Bosnia y Herzegovina pacíficamente entre Serbia y Croacia basándose en la etnicidad (ESI, 2019). Kupchan (2018) califica la propuesta serbokosovar de "limpieza étnica pacífica», y Rossi (2014: 871) teme que el intercambio de territorios provocaría una limpieza étnica del $60 \%$ de los serbokosovares que viven al sur del río Ibar (en municipios como Štrpce o Gračanica, que quedarían excluidos del acuerdo). Se abriría una «caja de Pandora» (Rossi, 2018a; Joseph, 2018), en una región donde las minorías son numerosas y muy repartidas en el territorio (Végh, 2012). Con relación al irredentismo albanés, las minorías más importantes se encuentran en Ulcinj (Montenegro) y el oeste de Macedonia (que estuvo al borde de una guerra civil en 2001, solucionada mediante los Acuerdos de Ohrid). Dérens (2007) recogió 
que el ministro de Asuntos Exteriores albanés, Besnik Mustafaj, ya declaró en 2006 que Albania pediría una revisión de todas sus fronteras en caso de una partición de Kosovo, y el primer ministro albanés, Edi Rama, expresó en 2018 la posibilidad de que hubiera un presidente único para Albania y Kosovo (DW, 19/02/2018). Respecto de los serbios, la cuestión de la Republika Srpska en Bosnia y Herzegovina es un conflicto latente, en el que Mirolad Dodik, su presidente, expresó que «si queremos unos Balcanes estables, las fronteras deben ser redibujadas» (Vijesti, 15/04/2019), a la vez que abogaba por un referéndum de autodeterminación e independencia. Vulin ya advirtió de que, si Bosnia y Herzegovina reconoce la independencia kosovar, apoyaría una Republika Srpska independiente (Ministarstvo Odbrane Republike Srbije, 17/09/2020). Recientemente, también se documentan protestas de los serbios en Montenegro (RFE/RL, 25/06/2020) ${ }^{12}$. Por todo ello, líderes de los Estados más sensibles y multiétnicos se han posicionado en contra de este acuerdo, como el primer ministro bosnio (Nezavisne, 30/08/2018) y Zoran Zaev, el primer ministro macedonio (N1, 31/01/2019).

Pese a todo, surge la duda de si los actores externos pueden vetar un acuerdo establecido por naciones soberanas. Es decir, una imposición paternalista a no llegar a un acuerdo por el temor de sus potenciales consecuencias negativas. Si ya se demostró en el pasado que las fronteras artificiales no consensuadas por los pueblos que atañen aportan más conflictos que beneficios, son los autóctonos los que deben establecer sus bases y sus acuerdos. Esta cuestión entronca plenamente con la legitimidad de los representantes y la calidad de la democracia. Es por ello que resulta necesario apuntar la negativa influencia que representa el auge de autoritarismos en los Balcanes (Bieber, 2020). Estos desvinculan a la ciudadanía del gobierno y fomentan un discurso nacionalista que puede repercutir en las relaciones interétnicas e internacionales. Es en este aspecto donde la UE puede ejercer gran influencia, mediante la acción del Comisario de Vecindad y Ampliación, para promover la mejora de la democracia en los Balcanes ${ }^{13}$. De igual manera, las últimas elecciones en Serbia han sido boicoteadas por todos los partidos de la oposición y ganadas por mayoría absoluta por Vučić, que ha contado con la felicitación de la UE. En lo que concierne a la corrección de fronteras, es fundamental prevenir un discurso autoritario y nacionalista y mejorar la situación de la democracia.

12. Las elecciones del 30 de agosto de 2020 en Montenegro resultaron con la formación de gobierno de la lista proserbia За будућност Црне Горе (Por el futuro de Montenegro), у fueron seguidas de incidentes contra los bosníacos en localidades como Pljevlja (Al Jazeera, 05/09/2020) y de consignas antikosovares (Radio Slobodna Evropa, 31/08/2020). También se organizaron manifestaciones promontenegrinas que coreaban «Nije ovo Srbija» (Esto no es Serbia) (Klix, 06/09/2020). La coalición de gobierno con otros partidos centristas se comprometió a seguir «la senda europea» y a no retirar el reconocimiento de la independencia kosovar.

13. En este sentido, la elección como comisario del húngaro Olivér Várhelyi (del partido de Viktor Orbán) fue criticada como contradictoria: el Gobierno de su partido, FIDESZ, ha sido advertido por el Parlamento Europeo de recibir sanciones como la pérdida del derecho a voto en el Consejo de la UE debido a su carácter «autocrático». 


\section{Conclusiones}

Las políticas de consociacionalismo en Kosovo han permitido aliviar la coexistencia de múltiples grupos étnicos, la paz, la mejora de la seguridad y la reducción de tensiones, y se han mostrado exitosas en la integración de las minorías, sobre todo en escenarios posconflicto. Sin embargo, el consociacionalismo también debilita la cohesión territorial del Estado y convierte las decisiones estatales en competiciones fragmentadas y multiescalares, al convertir este modelo de power-sharing en una etnocracia compartida, algo que puede observarse en la difícil gobernabilidad de Bosnia y Herzegovina y el deseo de independencia de la Republika Srpska, en consonancia de lo que señalan otros autores como Faludi (2013) o Bieber y Keil (2009). Como apunta Baliqi (2018), aunque en Kosovo ha logrado asegurar la paz y reducir las tensiones étnicas, también ha impedido la consolidación de la democracia y su statehood. Por ello es necesario basar más la democracia en actitudes cívicas y dirigir el voto según la propuesta más que en una primorosa etnicidad. La estabilidad política y la democratización del país son clave para permitir la reconciliación y aliviar las tensiones interétnicas, como también apunta Kelman (2005).

Desde una perspectiva teórica de la corrección de fronteras, y en un contexto de desfronterización-refronterización por la ampliación de la Unión Europea, y de predominio de las entidades supranacionales versus Estados nación, la propuesta de intercambio de territorios para corregir la frontera serbokosovar comporta aspectos positivos y negativos, y abre dudas sobre su idoneidad. En todo caso, resolver esta disputa es una labor necesaria, pertinente y que inevitablemente deberá realizarse de una u otra manera. Por ello, resulta esencial favorecer la democratización en sendos países, con tal de otorgar mayor legitimidad a un potencial acuerdo, que debe ser concertado entre los Estados implicados en un ejercicio de plena soberanía, el cual solo podría llevarse a cabo si son los habitantes de estas regiones quienes lo deciden y no las élites, como apunta Vulovi (2020) para los norkosovares. Algunos autores parecen mostrarse a favor de la propuesta, como Lasserre (2019), quien apunta que se podrían establecer acuerdos en materia de activos económicos o de protección de patrimonio, y siempre y cuando una vez realizado el intercambio se continuara con una política que siga garantizando los derechos de estas minorías para prevenir su discriminación y reducir su vulnerabilidad. Además, debe señalarse que las posibles repercusiones que origine estarían atenuadas por el hecho de que sería un intercambio consensuado y no unilateral.

La hipótesis de que los acuerdos fronterizos reducen la rivalidad interestatal presenta contradicciones según los estudios de caso y, en el que nos ocupa, levanta dudas sobre su idoneidad. Parece que la modificación de las fronteras para hacerlas coincidir con grupos étnicos presenta más riesgos que beneficios, debido a la existencia de minorías muy fragmentadas en los Balcanes y con latentes conflictos y aspiraciones de independencia que pueden hacer revivir los sucesos trágicos del pasado. Por ello, reconocer la independencia kosovar podría ser una resolución para dotar de legitimidad a sus autoridades y 
continuar con una supervisión internacional, como apunta Bargués-Pedreny (2016), además de avanzar en la democratización de la región. España se sitúa, junto con Chipre, en la posición más dura contra Kosovo, como Estado no reconocedor y que tampoco se ha comprometido a ninguna política con relación a Kosovo (Armakolas y Ker-Lindsay, 2020). Un eventual reconocimiento español podría traducirse en una mayor influencia en el seno de la UE y una mayor presencia de España en los Balcanes, que es casi inexistente.

En este sentido, debe prevenirse el ascenso de nacionalismos y autoritarismos, un papel en el que la UE puede exigir reformas de acuerdo con la negociación llevada a cabo entre los denominados Balcanes Occidentales y revisar su política exterior. Es necesario adoptar una postura común y asumir un papel central en el vecindario inmediato, y no propiciar situaciones de vacío que puedan ser aprovechadas por otros actores (EE. UU., Rusia, Turquía $)^{14}$. La UE debe ofrecer claridad y el compromiso firme y tangible de progreso para afianzar su credibilidad, pues lo contrario puede socavar el diálogo. Algunas de estas decisiones podrían comportar la liberalización de visados para kosovares y apoyar su independencia exigiendo la protección de las minorías como requisito clave para su acceso. Es necesario promover y garantizar que el bienestar está asegurado, independientemente de la etnia y el país de residencia. La gestión fronteriza y de reconocimiento entre Serbia y Kosovo es a la vez una oportunidad y un reto para la UE en su plano internacional, ya que puede servir como ejemplo para otros escenarios posconflicto o incluso en conflictos congelados. Si bien cada caso es singular y no se pueden establecer correlaciones directas, es una oportunidad para demostrar que la resolución de conflictos solo puede conseguirse mediante la credibilidad europea, la protección de las minorías y el fortalecimiento de la democracia.

\section{Referencias bibliográficas}

AitKen, Rob (2007). «Cementing divisions?». Policy Studies, 28 (3), 247-267. $<$ https://doi.org/10.1080/01442870701437568>

ANCEL, Jacques (2016) [orig. 1936]. «La geografía de las fronteras: el método». Geopolitica(s), 7 (1), 131-142. <https://doi.org/10.5209/rev_GEOP.2016.v7.n1.52954>

ARMAKOLAS, Ioannis y KER-LINDSAY, James (2020). The politics of recognition and engagement. EU Member State Relations with Kosovo. New Perspectives on SouthEast Europe. Cham, Suiza: Palgrave Macmillan. <https://doi.org/10.1007/978-3-030-17945-8>

14. La cumbre del 4 de septiembre de 2020 en Washington fue anunciada por los firmantes (Trump, Hoti, Vučić) como un «acontecimiento histórico», si bien únicamente se trataron asuntos económicos ya existentes. En materia de política internacional, solo comportó el reconocimiento de Israel a la independencia kosovar, el traslado de la embajada serbia a Jerusalén y el renombramiento del lago Gazivoda/Ujmani como lago Trump, lo cual generó más tensiones. La motivación parece responder más al deseo de buscar éxitos por parte de Trump (antes de las elecciones de noviembre de 2020), Hoti (por el desencanto popular por su gestión de la pandemia de COVID-19 tras la moción de censura contra Kurti) y Vučić (tras las controvertidas elecciones de junio de 2020). 
ASK (2012). Rezultatet përfundimtare të regjistrimit të popullsisë 2011. Pristina: Agjencioni i Statistikave të Kosovës.

BAKOTA, Ivica (2018). «Montenegro external relations briefing: Kosovo-Montenegro border demarcation problem and its implications for Montenegro». China-CEE Institute Weekly Briefing, 5 (4).

BALIQI, Bekim (2018). "Promoting multi-ethnicity or maintaining a divided society: dilemmas of power-sharing in Kosovo». Journal on Ethnopolitics and Minority Issues in Europe, 17 (1), 49-71.

BARgués-PEDrenY, Pol (2016). «From promoting to de-emphasizing "ethnicity": Rethinking the endless supervision of Kosovo». Journal of Intervention and Statebuilding, 10 (2), 222-240. <https://doi.org/10.1080/17502977.2015.1137392>

Bernabé-Crespo, Miguel Borja (2020). «Fronteras ocultas en la zona gris europea». Geopolitica(s). Revista de Estudios sobre Espacio y Poder, 11 (2), 71-93.

Bernabé-Crespo, Miguel Borja y PeÑA-Ramos, José Antonio (2019). «The management of water resources in a disputed border. The case of Gazivoda reservoir (Kosovo)». Fronteiras: Journal of Social, Technological and Environmental Science, 8 (1), 319-340. <https://doi.org/10.21664/2238-8869.2019v8i1.p319-340>

Bieber, Florian (2015). "The Serbia-Kosovo Agreements: An EU success story?». Review of Central and East European Law, 40 (3-4), 285-319. <https://doi.org/10.1163/15730352-04003008>

- (2017). "Trump and the Balkan Princes: What Trump's presidency means for South East Europe». London School of Economics Blog, 6 de febrero de 2017. Recuperado de <https://blogs.lse.ac.uk/europpblog/2017/02/06/trump-and-the-balkanprinces/>

- (2020). The rise of authoritarianism in the Western Balkans. New Perspectives on South East Europe. Palgrave Pivot. <https://doi.org/10.1007/978-3-030-22149-2>

BIEBER, Florian y KEIL, Sören (2009). «Power-sharing revisited: lessons learned in the Balkans». Review of Central and East European Law, 34, 337-360. <https://doi.org/10.1163/092598809X12474728805778>

BlitzT, Brad K. (2006). War and change in the Balkans. Nationalism, conflict and cooperation. Cambridge: Cambridge University Press. <https://doi.org/10.1177\%2F00223433070440040902>

BorGH, Chris van der (2012). "Resisting international state building in Kosovo». Problems of Post-Communism, 59 (2), 31-42. <https://doi.org/10.2753/PPC1075-8216590203>

BÜRKNER, Hans Joachim (2020). «Europeanisation versus Euroscepticism: Do borders matter?». Geopolitics, 25 (3), 545-566. $<$ https://doi.org/10.1080/14650045.2020.1723964>

CAIRO, Heriberto (2001). «Territorialidad y fronteras del estado-nación: Las condiciones de la política en un mundo fragmentado». Política y Sociedad, 36, 29-38. Recuperado de <https://revistas.ucm.es/index.php/POSO/article/view/ POSO0101130029A>.

Calić, Marie Janine (2000). «Kosovo in the twentieth century: A historical account». En: THAKUR, Ramesh y SCHNABEL, Albrecht (ed.). Kosovo and the challenge of humanitarian intervention: selective indignation, collective action, and international citizenship. Tokio: United Nations University Press. 
Calu, Marius Ionut (2020). Kosovo divided: Ethnicity, Nationalism and the Struggle for a State. Londres: I. B. Tauris. <http://dx.doi.org/10.5040/9781838606626>

CAPUsSELA, Andrea Lorenzo (2018). «Kosovo and Serbia: A dangerous but not unprecedented Balkan land swap». London School of Economics Blog, 29 de agosto de 2018. Recuperado de <https://blogs.lse.ac.uk/europpblog/2018/08/29/kosovoand-serbia-a-dangerous-but-not-unprecedented-balkan-land-swap/>

Constitución de la República de Kosovo (2008). Pristina: Parlamento de la República de Kosovo.

DAHLMAn, Carl T. (2017). «Faire frontière dans la paix: le Kosovo et la décentralisation de la concurrence ethno-politique». L'Espace Politique, 33. <https://doi.org/10.4000/espacepolitique.4424>

Dahlman, Carl T. y Ó TuATHAil, Gerard (2005). «The legacy of ethnic cleansing: The International Community and the returns process in Post-Dayton BosniaHerzegovina». Political Geography, 24 (5), 569-599. <https://doi.org/10.1016/j.polgeo.2005.01.007>

DemjaHA, Agon (2016). «Inter-ethnic relations in Kosovo». SEEU Review, 12 (1), 181-196. <https://doi.org/10.1515/seeur-2017-0013>

DÉREnS, Jean-Arnault (2007). "Kosovo: le piège va-t-il se refermer?». Politique Étrangère, 4, 713-725. <https://doi.org/10.3917/pe.074.0713>

DJUKANOVIć, Dragan (2008). «The post-conflict integration of minority ethnic communities in Kosovo». 3rd Annual Conference on Human Security, Terrorism and Organized Crime in the Western Balkan Region. Belgrado: HUMSEC.

ESI (2019). The Hypnotist: Aleksandar Vucic, John Bolton and the return of the past. Berlín, Bruselas, Estambul: European Stability Initiative Report, 25 de abril de 2019.

FABRY, Mikulas (2012). «The contemporary practice of state recognition: Kosovo, South Ossetia, Abkhazia, and their aftermath». Nationalities Papers, 40 (5), 661-676. <https://doi.org/10.1080/00905992.2012.705266>

FALUDI, Andreas (2013). "Territorial cohesion and subsidiarity under the European Union treaties: A critique of the "territorialism" underlying». Regional Studies, 47 (9), 1.594-1.606. <https://doi.org/10.1080/00343404.2012.657170>

FINLAY, Andrew (2011). Governing ethnic conflict. Consociation, identity and the price of peace. Londres: Routledge.

ForsberG, Tuomas y MäKInEN, Sirke (2019). "Russian discourse on borders and territorial questions - Crimea as a watershed?». Russian Politics, 4, 211-241. <https://doi.org/10.1163/2451-8921-00402004>

FORT, Emilie (2018). «From power-sharing arrangements to identity building: The case of Kosovo Serbs in Kosovo». Ethnopolitics, 17 (5), 503-518. <https://doi.org/10.1080/17449057.2018.1511159>

Gashi, Krenar; Musliu, Vjosa y Orbie, Jan (2017). «Mediation through recontextualization: The European Union and the dialogue between Kosovo and Serbia». European Foreign Affairs Review, 22 (4), 533-550. Recuperado de <http://hdl. handle.net/1854/LU-8554525>.

GiBAND, David y ViCENTE RUfí, Joan (2018). «Los espacios transfronterizos europeos: ¿un objeto geográfico de difícil definición? Una aproximación desde la perspectiva de los soft spaces». Documents d'Anàlisi Geogràfica, 64/3, 421-441. $<$ https://doi.org/10.5565/rev/dag.520> 
GuÉHEnNO, Jean-Marie (2000). The end of the Nation-State. Minneapolis: University of Minnesota Press.

Hartshorne, Richard (1937). «The Polish Corridor». Journal of Geography, 36, 161176.

Hopf, T. (2016). «Crimea is ours: a discursive history». International Relations, 30 (2), 227-255. <https://doi.org/10.1177\%2F0047117816645646>

Horowitz, Donald L. (1993). "Democracy in divided societies». Journal of Democracy, 4 (4), 18-38.

HuszkA, Beata (2007). «The Presevo Valley of Southern Serbia alongside Kosovo: The case for Decentralisation and Minority Protection». Centre for European Policy Studies, 120, 1-10.

ICJ (2010). Accordance with International Law of the Unilateral Declaration of Independance in Respect of Kosovo. International Court of Justice, Reports 2010, p. 403.

Islami, Hivzi y EJuPI, Arsim (2015). Popullsia dhe vendbanimet shqiptare të luginës së Preshevës (Albanian Population and settlements of the Presheva Valley: present situation and trends). Pristina: Kosova Academy of Sciences and Arts, Special Editions CXLVI. Section of Social Sciences, Book 50.

JEFFrey, Alex (2007). "The politics of 'democratization': Lessons from Bosnia and Iraq". Review of International Political Economy, 14 (3), 444-466. <https://doi.org/10.1080/09692290701395718>

Joseph, Edward P. (2018). «How to restart war in the Balkans». Foreign Policy, 4 de septiembre de 2018. Recuperado de <https://foreignpolicy.com/2018/09/04/howto-restart-war-in-the-balkans/>.

- (2020). «Anatomy of a Kosovo Summit Catastrophe». Foreign Policy, 24 de junio de 2020. Recuperado de <https://foreignpolicy.com/2020/06/24/kosovo-serbiasummit-white-house-catastrophe-balkans-peace-process $/>$.

KASA (2013). Kosova: A monographic survey. Pristina: Kosova Academy of Sciences and Arts.

KELMAN, Herbert C. (2005). «Building trust among enemies: the central challenge for internacional conflict resolution». International Journal of Intercultural Relations, 29, 639-650.

<https://doi.org/10.1016/j.ijintrel.2005.07.011>

Ker-LindSAY, James (2009). Kosovo. The path to contested statehood in the Balkans. Nueva York: IB Tauris.

- (2012). The Foreign Policy of Counter Secession: Preventing the Recognition of Contested States. Oxford: Oxford University Press.

KitANICS, Máté y PAP, Norbert (2012). «Las relaciones geopolíticas de las áreas albanesas». HAOL, 27, 103-116.

KRASNIQI, Gëzim (2012). «Overlapping jurisdictions, disputed territory, unsettled state: the perplexing case of citizenship in Kosovo». Citizenship Studies, 16 (3-4), 353-366.

<https://doi.org/10.1080/13621025.2012.683214>

Kupchan, Charles A. (2018). "An Offensive Plan for the Balkans That the U.S. Should Get Behind». The New York Times, de 13 de septiembre de 2018. Recuperado de <https://www.nytimes.com/2018/09/13/opinion/kosovo-serbia-landswap.html>.

Kymlicka, Will y STRaehle, Christine (2001). Cosmopolitismo, Estado-nación y nacionalismo de las minorías. México DF: Universidad Nacional Autónoma de México. 
LASSERRE, Frédéric (2019). "Le projet d'échange de territoires entre Serbie et Kosovo: une avenue crédible pour la paix?». Regards Géopolitiques, Bulletin du Conseil Québecois d'Études Géopolitiques, 5 (3), 25-40. Recuperado de <http://hdl.handle. net/20.500.11794/37253>.

LIJPHART, Arend (1977). Democracy in plural societies: A comparative exploration. New Haven: Yale University Press. $<$ https://doi.org/10.2307/1954827>

Malcolm, Noel (1998). Kosovo: a short history. Nueva York: New York University Press, Harper Perennial.

Marković, Ksenija; Jovanović, Marko y Matijević, Branka (2019). «Kosovo and Metohija or the European Union - A rhetorical dilemma in the Serbian political discourse». Serbian Political Thought, 66 (4), 173-192. <https://doi.org/10.22182/spm.6642019.8>

MARTíNEZ I EDO, Xavier (1993). «De la desintegración de la URSS a la recomposición del espacio ex-soviético». Boletín de la AGE, 17, 25-47.

Matveeva, Anna y PAes, Wolf Christian (2003). The Kosovo Serbs: An ethnic minority between collaboration and defiance. Bonn: BIIC.

MoOre, Margaret (1998). National self-determination and secession. Oxford: Oxford University Press. <https://doi.org/10.1093/0198293844.001.0001>

Murphy, Alexander B. (1990). «Historical justifications for territorial claims». Annals of the Association of American Geographers, 80 (4), 531-548. <https://doi.org/10.1111/j.1467-8306.1990.tb00316.x>

Oswiak, Andrew P. y Rider, Toby J. (2013). "Clearing the hurdle: Border settlement and rivalry termination». The Journal of Politics, 75 (3), 757-772. <https://doi.org/10.1017/S0022381613000595>

RAMET, Sabrina P. (2002). Balkan Babel: The disintegration of Yugoslavia from the death of Tito to the fall of Milosevic. Boulder: Westview Press.

RATZEL, Friedrich (1896). «Die Gesetze des räumlichen Wachstums der Staaten». Peterman Mitteilugen, 42, 97-107.

Rossi, Michael (2014). «Ending the impasse in Kosovo: partition, decentralization, or consociationalism?». Nationalities Papers, 42 (5), 867-889. <https://doi.org/10.1080/00905992.2014.937683>

- (2018a). «Partition in Kosovo will lead to disaster». Foreign Policy, 19 de septiembre de 2018. Recuperado de <https:/foreignpolicy.com/2018/09/19/partition-inkosovo-will-lead-to-disaster-serbia-vucic-thaci-mitrovica-ibar/>.

- (2018b). «A land swap between Kosovo and Serbia would be deeply problematic and potentially dangerous». London School of Economics Blog, 17 de agosto de 2018. Recuperado de <https://blogs.lse.ac.uk/europpblog/2018/08/17/a-land-swap-between-kosovo-and-serbia-would-be-deeply-problematic-and-potentially-dangerous/>.

RoTHCHILD, Donald S. y LAKE, David A. (2005). «Territorial decentralization and civil war settlements». En: Roeder, Philip G. y Rothchild, Donald S. (eds.). Sustainable Peace: Power and democracy after civil wars. Nueva York: Cornell University Press, 109-132.

Roux, Michel (2003). "Controverses sur les frontières du Kosovo». Balkanologie. Revue d'Études Pluridisciplinaires, 7 (2), 183-197.

Simmons, Beth A. (2005). «Rules over Real Estate: Trade, territorial conflict and international borders as institution». The Journal of Conflict Resolution, 49 (6), 823-848. Recuperado de <https://ssrn.com/abstract=1518016>. 
SpaHiU, Nexhmedin (1999). «Serb proposals for partitioning Kosova». Central Europe Review, 1 (1).

TOKTOGulOv, Beishenbek (2018). «The failure of settlement on Kyrgyz-Uzbek border ussues: a lack of diplomacy?». Bilge Strateji, 10 (19), 85-106.

VÉGH, Andor (2012). "Minorities, mother countries, majority on the Western Balkans». HAOL, 27, 83-101.

VelázQuez, César A. y Pérez, Gabriel (2010). «Las transformaciones del Estadonación en el contexto de la globalización». Política y Cultura, 34, 107-127. Recuperado de <http://www.scielo.org.mx/scielo.php?script=sci_arttext\&pid=S0188$77422010000200006 \&$ lng=es\&tlng=es $>$.

VladisAVlJEVIĆ, Nebojsa (2004). "Grassroots groups, Milosevic or dissident intellectuals? A controversy over the origins and dynamics of the mobilisation of Kosovo Serbs in the 1980s». Nationalities Papers, 32 (4), 783-796. <https://doi.org/10.1080/0090599042000296113>

Vulović, Marina (2020). «Performing statehood in Northern Kosovo: Discursive struggle over contested space». Cooperation and Conflict, OnlineFirst. <https://doi.org/10.1177\%2F0010836720906851>

Waters, Timothy William (2020). Boxing Pandora: Rethinking borders, States and Secession in a democratic world. New Haven y Londres: Yale University Press.

ZACHER, M. (2001). «The territorial integrity norm: International boundaries and the use of force». International Organization, 55 (2), 215-250.

Zejnullahi, Veton (2015). «Albanians in Presevo Valley and their national rights». European Journal of Language and Literature Studies, 2 (1), 89-94.

<https://dx.doi.org/10.26417/ejls.v2i1.p90-99>

\section{Noticias de prensa}

Al JazeERA (05/06/2020). "Bosniaks in Montenegro live in fear, anxiety following election». <https://www.aljazeera.com/news/2020/09/05/bosniaks-in-montenegrolive-in-fear-anxiety-following-election/>.

B92 (21/04/2017). «Greater Albania must include Presevo, and Nis, says mayor». <https:/www.b92.net/eng/news/politics.php?.yyy=2017\&mm=04\&dd=21\&nav_ $\mathrm{id}=101083>$.

- (09/08/2018). «Vucic: My policy is delimitation with Albanians». <https://www. b92.net/eng/news/politics.php? yyyy=2018\&mm=08\&dd=09\&nav_id=104824>.

- (27/05/2020). "Mustafa: The final phase of the dialogue between Serbia and Kosovo soon to follow». <https://www.b92.net/eng/news/politics. php? yyyy=2020\&mm=05\&dd=27\&nav_id=108569>.

- (31/05/2020). "Vucic: There's no doubt - without resolving Kosovo issue, Serbia will not join the EU». <https://www.b92.net/eng/news/politics. php? yyyy=2020\&mm=05\&dd=31\&nav_id=108594>.

- (11/06/2020). "Vucic: The recognition of Kosovo is not on the agenda». <https://www. b92.net/eng/news/politics.php?yyy=2020\&mm=06\&dd=11\&nav_id=108668>.

BeTA (15/06/2020). «Putin: Nijedna odluka o Kosovu ne može biti doneta bez Saveta bezbednosti UN». <https://beta.rs/vesti/politika-vesti-srbija/128884-putin-nijedna-odluka-o-kosovu-ne-moze-biti-doneta-bez-saveta-bezbednosti-un>.

BLIC (28/05/2018). «Dačić: Podela Kosova jedino moguće i brzo rešenje, ali treba videti i druge opcije». <https://www.blic.rs/vesti/politika/dacic-podela-kosovajedino-moguce-i-brzo-resenje-ali-treba-videti-i-druge-opcije/19pefsm>. 
- (25/07/2018). «Vučić: Nećete razgraničenje sa Albancima? A za 40 godina ćemo braniti Vranje...». <https://www.blic.rs/vesti/politika/vucic-necete-razgranicenjesa-albancima-a-za-40-godina-cemo-braniti-vranje/0zrjg64>.

COURRIER DES BALKANS (03/06/2020). «Kosovo: 168.000 euros pour promouvoir l'idée d'un échange de territoires». <https://www.courrierdesbalkans.fr/Kosovofait-la-promotion-de-l-echange-de-territoires $>$.

DANAS (04/03/2019). "Vučić nagovestio da ne isključuje mogućnost priznanja Kosova». <https://www.danas.rs/politika/vucic-nagovestio-da-ne-iskljucuje-mogucnostpriznanja-kosova/>.

DW (19/02/2018). "Albania's Edi Rama floats joint president idea in Kosovo». $<$ https://www.dw.com/en/albanias-edi-rama-floats-joint-president-idea-inkosovo/a-42642058>.

Europa Press (14/05/2020). «El enviado de la UE para el diálogo Belgrado-Pristina afirma que el canje de territorios no está en la agenda». <https://www.europapress. es/internacional/noticia-enviado-ue-dialogo-belgrado-pristina-afirma-canje-territorios-no-agenda-20200514182128.html>.

Glas Amerike (28/03/2008). «Bolton: Podela Kosova bila bi pravična, ali ne bi rešila problem manjina». <https://www.glasamerike.net/a/a-34-2008-03-28voa6-86820287/741736.html>.

IBNA (25/02/2019). «The detailed agreement between Kosovo and Serbia is leaked». $<$ https://balkaneu.com/the-detailed-agreement-between-kosovo-and-serbia-isleaked/>.

KLIX (06/09/2020). «Hiljade ljudi na skupu u Podgorici: Ne damo Crnu Goru, ne smije biti crnih ptica ni Srebrenica». <https:/www.klix.ba/vijesti/regija/hiljadeljudi-na-skupu-u-podgorici-ne-damo-crnu-goru-ne-smije-biti-crnih-ptica-nisrebrenica/200906089>.

Kosovo 2.0 (17/02/2019). «Voices from the borderlands». <https://kosovotwopointzero.com/en/voices-from-the-borderlands/>.

Kossev (06/02/2018). «Unutrašnji dijalog o KiM: Adresa za rešenje srpsko-albanskog sukoba - Tirana, ne Priština». <https://kossev.info/unutrasnji-dijalog-o-kimadresa-za-resenje-srpsko-albanskog-sukoba-tirana-ne-pristina/>.

- (22/09/2018). «Koha: Thaci will return Rankovic's gift to Serbia». <https://kossev. info/koha-thaci-will-return-rankovics-gift-to-serbia/>.

KSC (24/06/2020). "Kosovo Specialist Chambers \& Specialist Prosecutor's Office - Press Statement 24 June 2020». <https://www.scp-ks.org/en/pressstatement>.

Ministarstvo Odbrane Republike Srbije (17/09/2020). «Ministar Vulin: Ako su u BiH za nezavisno "Kosovo", ja sam za nezavisnu Republiku Srpsku». <http:// www.mod.gov.rs/lat/16493/ministar-vulin-ako-su-u-bih-za-nezavisno-kosovo-jasam-za-nezavisnu-republiku-srpsku-16493>.

N1 (31/01/2019). «Macedonia wants positive solution for Kosovo, Zaev tells N1». <http://rs.n1 info.com/English/NEWS/a456419/Macedonia-wants-positive-solution-for-Kosovo-Zaev-tells-N1.html>.

- (05/02/2019). «Kosovo President: Minor border correction acceptable for deal with Belgrade». <http://rs.n1info.com/English/NEWS/a458057/Kosovo-s-Thacisays-small-border-correction-with-Serbia-possible.html>.

National Review (28/03/2019). «Pompeo Casts Golan Heights Move As a Rejection of Conventional Wisdom». <https://www.nationalreview.com/news/mikepompeo-recognition-israel-sovereignty-golan-heights/>. 
NeZAVISNE (27/03/2011). «Tadić: Albanci neće Srbiju, Srbi neće Kosovo». <https:// www.nezavisne.com/novosti/ex-yu/Tadic-Albanci-nece-Srbiju-Srbi-neceKosovo/84098>.

- (30/08/2018). "Zvizdić: Priča o promjeni granica opasna». <https://www.nezavisne.com/novosti/bih/Zvizdic-Prica-o-promjeni-granica-opasna/495037>.

President of The Republic of Kosovo (08/01/2019). "President Thaçi writes to President Trump, pledges to work towards a historic agreement with Serbia». $<$ https://president-ksgov.net/en/news/president-thaci-writes-to-president-trumppledges-to-work-towards-a-historic-agreement-with-serbia>.

PRISTINA INSIGHT (28/05/2020). «Border changes promoted with taxpayer money». $<$ https://prishtinainsight.com/border-changes-promoted-with-taxpayer-money/>.

- (09/06/2020). «More land swap lobbying uncovered». <https://prishtinainsight. com/more-land-swap-lobbying-uncovered/>.

Radio Slobodna Evropa (31/08/2020). "Crna Gora: Demokratski front slavi izborni rezultat pjesmama o Kosovu». <https://www.slobodnaevropa.org/a/30811825. html? nocache $=1>$.

RFE/RL (19/06/2020). «Moscow’s Approval Necessary For Any Deal Between Serbia, Kosovo, Lavrov Says». <https:/www.rferl.org/a/russia-serbia-kosovo-lavrovgrenell/30679106.html>.

- (25/06/2020). «Police In Montenegro Detain Dozens After Pro-Serbian Protests». <https://www.rferl.org/a/police-in-montenegro-detain-dozens-after-pro-serbprotests/30690097.html>.

RTS (16/08/2018). "Vučić o KiM: Ako bude sporazuma, imaćemo više nego danas». <https://www.rts.rs/page/stories/sr/story/9/politika/3231538/vucic-o-kim-akobude-sporazuma-imacemo-vise-nego-danas.html>.

- (02/03/2019). "Vučić za RTS: Priština čeka povoljan trenutak za napad na sever». <https:/www.rts.rs/page/stories/sr/story/9/politika/3438739/vucic-za-rts-pristinaceka-povoljan-trenutak-za-napad-na-seve.html>.

TelegraF (15/09/2020). «Danas se prvi put u istoriji obeležava Dan srpskog jedinstva: Slave ga Srbija i Republika Srpska». <https://www.telegraf.rs/vesti/srbija/3237505danas-se-prvi-put-u-istoriji-obelezava-dan-srpskog-jedinstva-slave-ga-srbija-i-republika-srpska>.

The IndEPENDENT (21/03/2018). «Tear gas released in Kosovo parliament by opposition party in bid to stop vote». <https:/www.independent.co.uk/news/world/ europe/kosovo-parliament-tear-gas-opposition-party-stop-vote-vetevendosje-montenegro-a8266441.html>.

ViJESTI (15/04/2019). "Dodik: Da nema stranaca BiH ne bi trajala ni dva dana». <https://vijesti.ba/clanak/442960/dodik-da-nema-stranaca-bih-ne-bi-trajala-nidva-dana>.

- (26/09/2020) «Vulin: Vučić je predsjednik svih Srba». <https://vijesti.ba/clanak/508539/vulin-vucic-je-predsjednik-svih-srba>. 